\title{
Control of FES-induced cyclical movements of the lower leg
}

\author{
P. H. Veltink
}

Biomedical Enginearing Division, Department of Electrical Engineering, University of T Wente PO Box 217. 7500 AE Enschede, The Netherlands

\begin{abstract}
The control of a cyclical movement of the lower leg with electrical stimulation of the quadriceps muscles is formulated as an optimal control problem. The time integral of knee torque is taken as the optimisation criterion. As an additional condition, every cycle a certain reference maximum angle should be reached. A model study indicates that one stimulation burst per cycle at the maximum recruitment level is a suboptimal solution to this problem. To compensate for the influence of muscle fatigue, the bust time is adaptively adjusted by a discrete time PID-controller on the basis of the performance in the previous cycles. This strategy appeared to be successful in experimental tests. A considerable time difference (about $0.15 \mathrm{~s}$ ) was found between the end of the stimulation burst and the tracking of the passive state trajectory, which satisfies the maximum angle condition.
\end{abstract}

Keywords-Electrical stimulation, Optimal control, PID-control

Med. 8 Biol Eng. 8 Comput. 1991, 29, NS8 NS12

\section{Introduction}

WALKING is a cyclical movement. This should be taken into account when walking is generated in paraplegics using FES. Patterns for stimulation of the muscles have to be identified as a function of the time within each cycle (MCNeAl et al., 1989; MARSOLAIS and KoBETIC, 1983; CHIZECK et al., 1988). The open loop use of these patterns can result in a good walking pattern as long as the muscles do not fatigue and no external disturbances are present.

What strategy can be used to derive the stimulation patterns? One possibility is to determine the stimulation patterns that will result in a prescribed trajectory of the paralysed leg. MCNeal et al. (1989) did this for only a freely swinging lower leg. The trajectory of a healthy subject could be taken as the reference trajectory. However, this is not necessarily optimal for a patient who walks with an artificial system with only a few muscles stimulated, and possibly using mechanical orthoses and walking aids.

Another approach is to find a stimulation sequence that will maximise an optimisation criterion, which is, for example, related to minimum energy consumption of the stimulated muscle (YAMAGUCHI and ZAJAC, 1990). This criterion should be optimised under the restrictions of certain conditions which are related to the desired movement, e.g. a given step length, walking speed etc.

In this paper we will investigate this approach for the simple problem of controlling a freely swinging lower leg by electrical stimulation of the quadriceps muscles. Furthermore, strategies will be discussed for adjusting stimulation parameters when muscle fatigue influences the system response.

Received 13th February 1991

(C) IFMBE: 1991

\section{Suboptimal control of the cyclical movement of the lower leg}

The system considered in this paper is a freely swinging lower leg (Fig. 1). A cyclical movement is studied, reaching a certain maximum reference angle $\phi_{\max \text { ref }}$ every cycle. The movement is generated by artifical transcutaneous electrical stimulation of the quadriceps muscles. The objective is to generate this movement with minimum time integral of the actively developed knee torque in a cycle:

$$
C=\int_{0}^{T_{\mathrm{eyc}}}|M(t)| d t
$$

This criterion is related to the energy consumption of the muscle (CROWNINSHIELD and BRAND, 1981).

In a model study of the system, we estimated a suboptimal stimulation pattern, assuming an ideal torque generator at the knee joint, generating one rectangular torque pulse per cycle in response to a stimulaton burst applied to the quadriceps muscles at constant recruitment level and stimulation frequency.

The swinging lower leg can be described by a pendulum, resulting in a second order nonlinear model of the biomechanical system:

$$
M_{\text {quad }}=I \ddot{\phi}+D \dot{\phi}+K_{1} \sin \phi+K_{2}(\phi)
$$

The moment of inertia $I$ and the damping $D$ are assumed to be linear. The nonlinear 'stiffness' component consists of two parts: the contribution of gravity, described by the sine term and the internal joint stiffness, described by the function $K_{2}(\phi)$. The internal joint stiffness becomes large near full knee extension, but is small in the angle range we considered for the cyclical leg movement. In this range the gravity contribution is dominant. In the following analysis we will assume the stiffness to be linear, and therefore will 
approximate the total 'stiffness' component by a term $K \phi$. Under this approximation, the system is a second order linear system, which can be described by a natural frequency $\omega_{n}$, a damping ratio $\zeta$ and a stiffness $K$. The lower leg is a subcritically damped system $(\zeta<1)$. The impulse response is a damped sine signal:

$$
h(t)=\frac{\omega_{n} e^{-\zeta \omega_{n} t}}{K \sqrt{ }\left(1-\zeta^{2}\right)} \sin \left[\omega_{n} \sqrt{ }\left(1-\zeta^{2}\right) t\right] \quad t>=0
$$

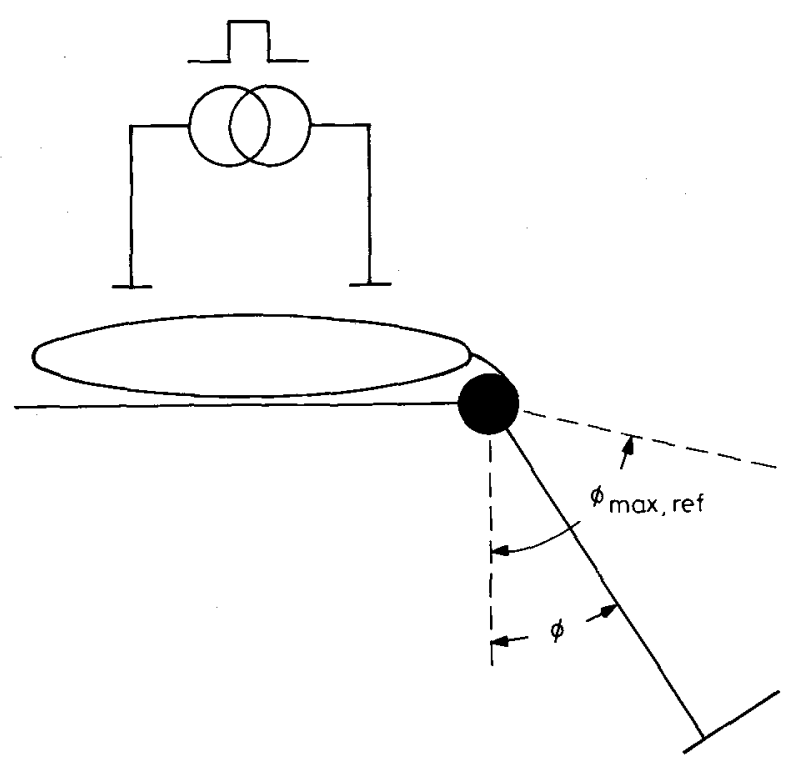

Fig. 1 Schematic drawing of a freely swinging lower leg with stimulation of the quadriceps muscles. In this study of cyclical lower leg movements, the objective was to reach the reference maximum knee angle $\phi_{\max \text { ref }}$ every cycle, while minimising a criterion which is related to energy consumption of the muscle

\section{Closed-loop adaptation of stimulus burst duration}

When the muscle fatigues, the response to a stimulation burst will change. To compensate for this changing system response, the stimulation burst time $T_{o n}$ has to be increased such that $\varphi_{\max r e f}$ is reached every cycle.

\subsection{Discrete-time PID-controller}

Such an adaptation of the timing of the stimulation sequence can be achieved by a closed-loop controller, such as a discrete-time PID-controller which adjusts the burst time $T_{o n}$ on the basis of differences between the maximum angle $\phi_{\max }$ and the reference angle $\phi_{\max \text { ref }}$ in the previous cycles (Fig. 2). The time step of this discrete-time PIDcontroller is the cycle time $T_{c y c}$.

The PID-controller can be tuned on the basis of a rootlocus analysis of the total system (PHILLIPs and NAGLE, 1984). The swinging lower leg is described by the impulse response of eqn. 3. When tuning the PID-controller, this impulse response has to be expressed in discrete time, with a time step equal to the cycle time. From eqn. 3, an expression in discrete time can be derived when considering only

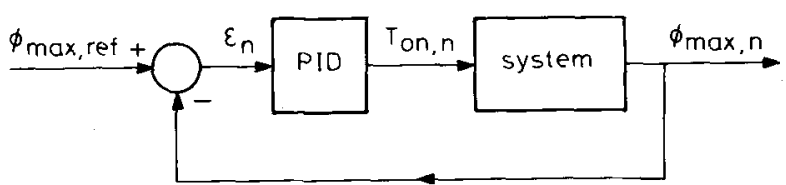

Fig. 2 Discrete-time PID-controller for adjusting the timing parameter $T_{o n}$ of the stimulation sequence used for the cyclical movement the maximum every cycle:

$$
\phi_{\max n}=b a^{n}
$$

$b$ and $a$ are parameters. Parameter $a$ is described by

$$
a=e^{-2 \pi\left(\zeta / \sqrt{ }\left(1-\zeta^{2}\right)\right)}
$$

The $z$-transform of this impulse response is

$$
\phi_{\max }(z)=\frac{b z}{z-a}
$$

The system can therefore be represented by a pole at $z=a$ and a zero at $z=0$.

In the $z$-domain, the discrete time PID-controller is given by (PHILliPs and NAGLe, 1984)

$$
\operatorname{PID}(z)=\frac{T_{o n}(z)}{\varepsilon(z)}=\frac{G\left(z-z_{1}\right)\left(z-z_{2}\right)}{z(z-1)}
$$

The corresponding discrete-time algorithm for the PIDcontroller is

$$
T_{o n n}=T_{o n n-1}+G\left(\varepsilon_{n}-\left(z_{1}+z_{2}\right) \varepsilon_{n-1}+z_{1} z_{2} \varepsilon_{n-2}\right)
$$

The zeros $z_{1}$ and $z_{2}$ and the gain $G$ have to be determined when tuning the PID-controller.

\subsection{Feasibility of controlling the stimulation during the cycles on the basis of real-time measurement of the state variables}

Control of the stimulation burst time during the cycles, on the basis of real-time measurement of the state variables of the system, could be an altnernative to the proposed control strategy on the basis of errors in the maximum angles in the previous cycles. The feasibility of such an approach depends on the time delays between the stimulation burst and the influences of the stimulation on the resulting movement. These time delays can be evaluated in state space.

The state variables of the second order pendulum system are knee angle $\phi$ and knee angular velocity $\phi$. The state of the system at a given moment in time can be represented by a point in the state space spanned by the knee angle and knee angular velocity. The maximum angle condition will be met if the knee torque is zero after the state of the system crosses the trajectory of the passive biomechanical system which reaches the maximum reference angle $\phi_{\max \text { ref }}$ at zero angular velocity. However, there is a time difference between the end of the stimulus burst and the moment the passive state trajectory is crossed, due to the time delay between stimulation and muscle activation and to the relatively slow muscle dynamics. Therefore, the system performance has to be predicted over this time delay to stop stimulation at the correct moment. A model of the muscle dynamics and knowledge of the present muscle fatigue is needed for this prediction.

\section{Methods}

4.1 A model study of suboptimal control of the cyclical lower leg movements

One cycle of the lower leg movement starting from the same initial state was simulated using the system model of eqn. 2. The input of the system was a rectangular torque pulse. The width and amplitude of this torque pulse was varied in such a way that the torque-time integral over the 
cycle (eqn. 1) was constant. We evaluated which burst width would result in the highest maximum angle.

\subsection{Preliminary experimental test of the PID-controller}

In three healthy subjects a preliminary experimental evaluation of the PID-controlled cyclical movement was performed. The subjects were sitting on a bench and were instructed to relax as much as possible during the experiment. Their lower legs could swing freely. The knee angle was measured with a goniometer. The quadriceps muscles of one leg were stimulated using two electrodes on the skin above this muscle group. The pulse width was $300 \mu \mathrm{s}$ and the stimulation frequency was $20 \mathrm{~Hz}$. In the healthy subjects we could not increase the stimulation amplitude to a level of maximum recruitment. Therefore, the recruitment level stayed submaximum. The pulse amplitude $A$ was between 40 and $50 \mu \mathrm{A}$. The stimulator was coupled to a PC-AT compatible computer, on which the controller was implemented. The knee angle was sampled at $100 \mathrm{~Hz}$ using an Analogue Devices RTI815 A/D board. The knee angle was filtered using a moving average filter with a width of five samples.

The protocol of the experiment was as follows:

(i) First, the response of the passively swinging lower leg was determined. The lower leg was first lifted by the experimenter, and then released. From this experiment the pole ' $a$ ' (eqn. 6) was determined as the quotient between subsequent maxima of the damped nearsinusoidal movement. Also the cycle time $T_{c y c}$ was determined.

(ii) The PID-controller was tuned: we placed one zero at the system pole ' $a$ ', and the other at $z=0.2$ (root locus analysis). The step responses of the system with PID-controlled adaptation of the stimulation burst width $T_{o n}$ were determined, and the gain $G$ of the PIDcontroller was varied until the speed and overshoot of the response were satisfactory.

(iii) The performance of the PID-controller in realising $\phi_{\max \text { ref }}$ every cycle over a time period of $2.5 \mathrm{~min}$ was evaluated. The cycle time was taken to be the same as the cycle time $T_{c y c}$ of the passively swinging lower leg.

(iv) After a period of $15 \mathrm{~min}$ rest, a $2.5 \mathrm{~min}$ open-loop cyclical movement was carried out. The burst time $T_{o n}$ was held constant. The maximum angle $\phi_{\max }$ as a function of the cycle number was evaluated and compared with the closed-loop result.

\section{Results}

\subsection{Modelling results on suboptimal control of the cyclical} lower leg movement

Fig. 3 shows the simulation results in state space for one cycle of a leg movement with varying burst width $T_{o n}$ and constant torque-time integral (eqn. 1). The damping constant $\zeta=0 \cdot 1$ corresponds to a pole ' $a$ ' of $0 \cdot 53$. The conclusion from this simulation is that, for the damping constant and burst widths considered, the highest maximum angle occurs at the shortest burst width, keeping the time integral of the torque over a cycle constant. Therefore, when a prescribed maximum reference angle $\phi_{\max r e f}$ is to be realised with a rectangular torque pulse having the smallest time integral, the amplitude of the torque pulse should be maximum and, consequently, its width should be minimum.

When knee torque is generated by electrical stimulation of the quadriceps, a rectangular torque pulse cannot be realised because of the muscle dynamics. Therefore, the

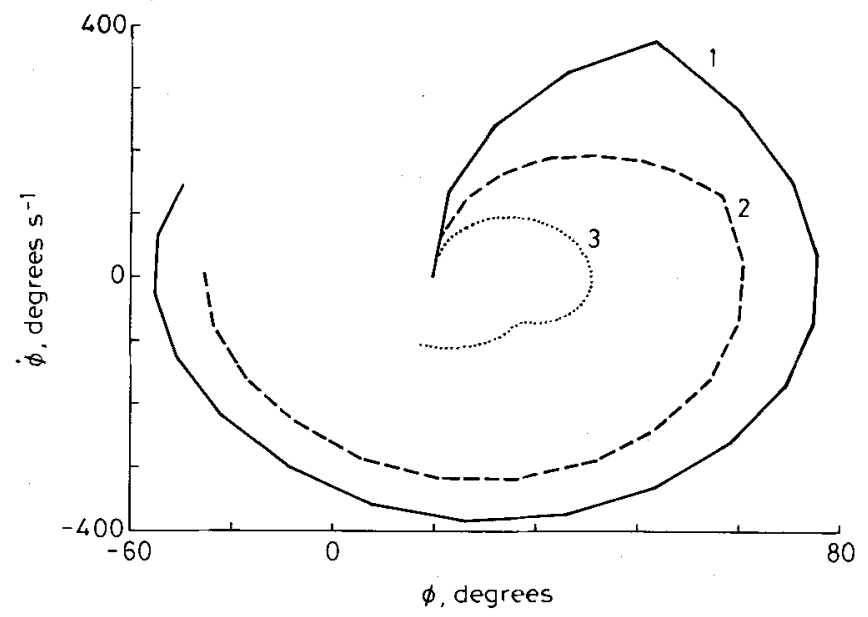

Fig. 3 Simulated response in state space of one cycle of the lower leg movement starting at $\phi=0$ and $\dot{\phi}=0$ for three widths of the stimulation burst, and constant torque-time integral

$$
\begin{array}{ll}
1 & T_{\text {on }}=0.4 \mathrm{~s} ; M_{\text {burst }} / K=1.2 \mathrm{rad} \\
2 & T_{o n}=0.8 \mathrm{~s} ; M_{\text {burst }} / K=0.6 \mathrm{rad} \\
3 & T_{o n}=1.6 \mathrm{~s} ; M_{\text {burst }} / K=0.3 \mathrm{rad} \\
& \omega_{n}=2 \pi \mathrm{rad} \mathrm{s}^{-1} ; \zeta=0.1
\end{array}
$$

Points in the state space responses lie $0.05 \mathrm{~s}$ apart, which corresponds to a stimulus period at $20 \mathrm{~Hz}$ stimulation. The calculation time step was $5 \mathrm{~ms}(10$ time steps per stimulus period)

maximum stimulation of the quadriceps in one burst per cycle might be a suboptimal control strategy.

\subsection{Preliminary experimental test of the PID-control of the cyclical movement}

An example of the passive lower leg response is given in Fig. 4 . The average value of ' $a$ ' was about $0 \cdot 6$, which corresponds to $\zeta \simeq 0.08$. However, the quotient of subsequent maxima tended to first increase, and subsequently decrease with the number of the maximum. This points to a nonlinearity in the system. The cycle time was $1.0 \mathrm{~s}$. These parameters corresponded well in all three subjects.

The results of closed- and open-loop controlled cyclical

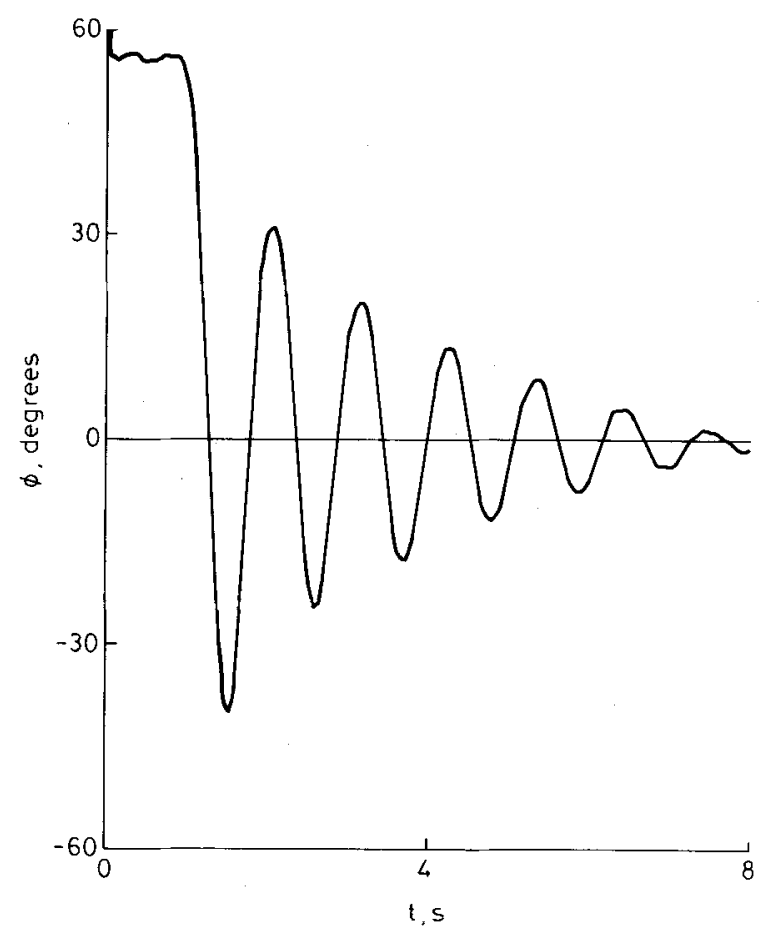

Fig. 4 An example of the passive lower leg response 
movements over 150 cycles are shown in Fig. 5. The PIDcontroller appears to be able to realise the reference angle $\phi_{\text {max } r e f}$ over the 150 cycles, despite the coarse variation of the limited number of stimulation pulses ( $3-5$ pulses per cycle). Especially the switch from 4 to 5 pulses per cycle can be seen in the response of the maximum angle $\phi_{\max }$.
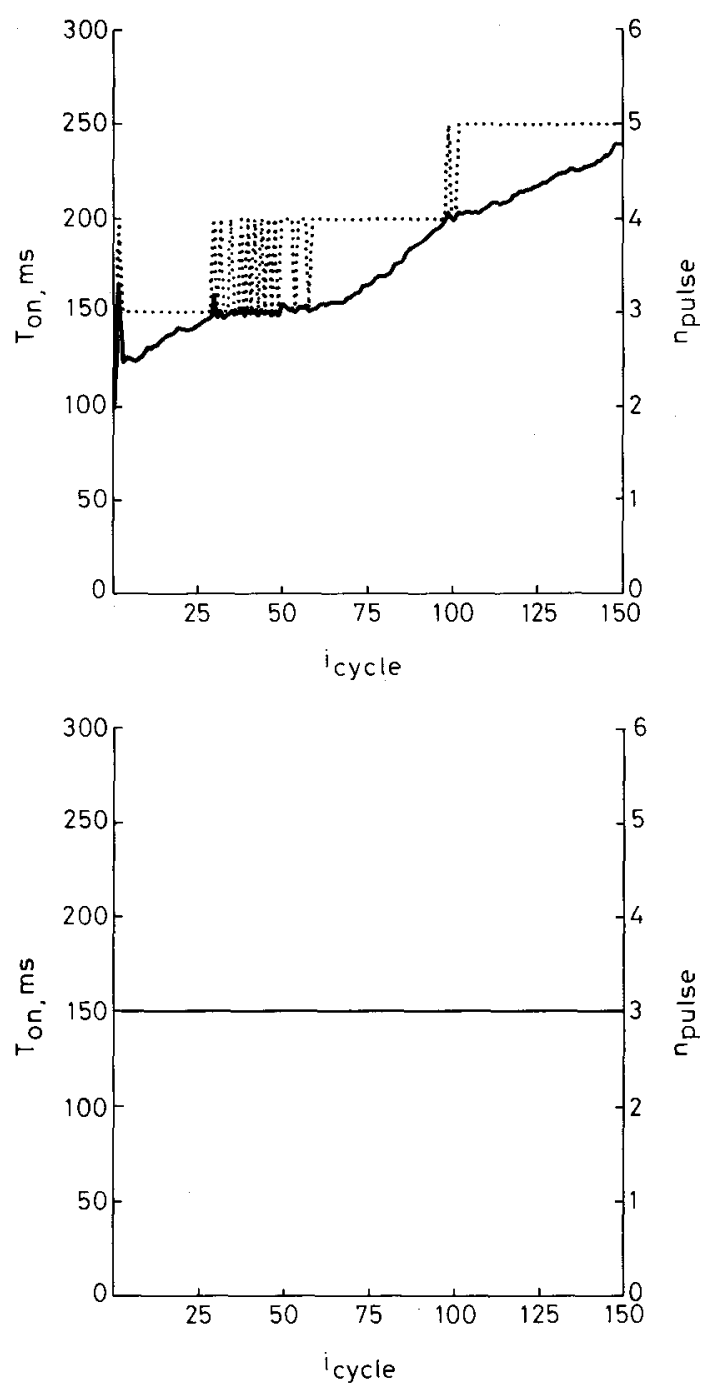

although some variation from cycle to cycle remained. It appeared to be important to use the exact cycle time $T_{c y c}$, as estimated from the passive response. When the cycle time was taken to be $0.05 \mathrm{~s}$ longer or shorter, cycle to cycle variations in the maximum angle increased, because the muscle is not activated in the correct phase of the cycle.
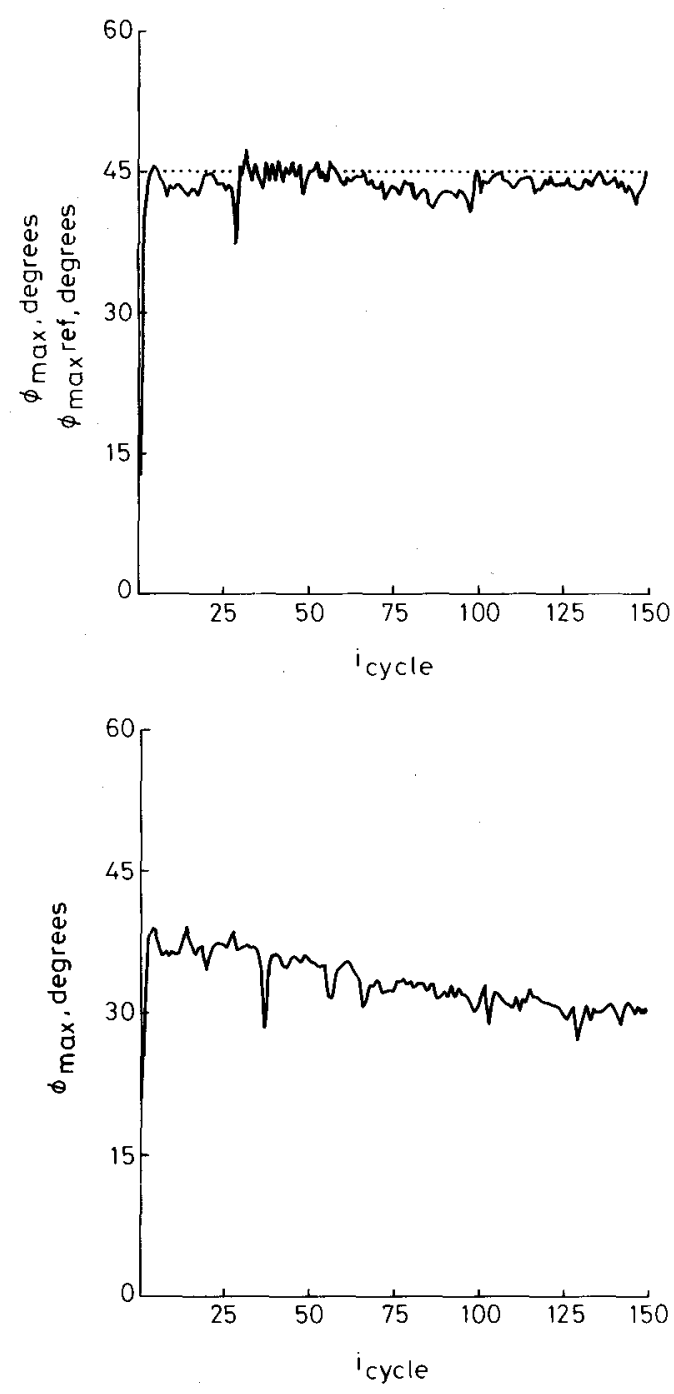

Fig. $5 T_{o n}, n_{\text {pulse }}$ and $\phi_{\max }$ as a function of cycle number $i_{\text {cvcle }}$ during 150 cycles of closed and open loop controlled cyclical lower leg movements. (a) closed loop controlled movements $\left(z_{1}=0 \cdot 2, z_{2}=0 \cdot 6, G=3 \mathrm{~ms} \mathrm{deg}^{-1}\right)$; (b) open loop controlled movements $\left(T_{o n}=0.15 \mathrm{~s} ; n_{\text {pulse }}=3\right)$. Left recording: $T_{o n}$ (solid line) and the number of stimulation pulse $n_{\text {pulse }}$ of the burst in each cycle (dotted line). Right recording: $\phi_{\max }$ (solid line) and $\phi_{\max \text { ref }}$ (dotted line: only in Fig. 5a)

The remaining cycle to cycle variations of the $\phi_{\max }$ values may be due to variations of the system response. For two of the three persons, fatigue occurred during these 150 cycles. The PID-controller increased $T_{o n}$ to compensate for this fatigue (Fig. 5a). In the open loop experiment (Fig. 5b) $\phi_{\max }$ decreased during the 150 cycles for both persons showing fatigue. As can be seen in Fig. 5, the decrease of $\phi_{\max }$ in the open loop trial was not as fast as the increase in burst width $T_{o n}$ for compensation of fatigue during the PID-controller trial. This corresponds to the observation in the simulation study that shorter stimulation bursts are more efficient than longer bursts. In one of the two persons showing fatigue in the 150 cycles, the trial for the PIDcontroller was extended to 300 cycles in total. In the last cycles the maximum angle could no longer be realised because of the limited range of the system response. This resulted in a steady fast increase of burst width, which was no longer effective. The third subject did not show fatigue during the 150 cycles, and therefore $T_{o n}$ did not increase to compensate fatigue. Also in this person, the PID-controller was able to realise the reference maximum angle $\phi_{\max r e f}$,

\subsection{Time delays between stimulation burst and state space trajectory tracking}

Fig. $6 a$ shows the knee angle $\phi$, knee angular velocity $\phi$ and stimulus amplitude $A$ as a function of time for three cycles of the PID-controller trial of Fig. $5 a$. Fig. $6 b$ shows the same registrations in state space. The spiral trajectory in this figure corresponds to the passive response of a linear second order system $(\zeta=0.08)$, reaching $\phi_{\max r e f}$ at $\dot{\phi}=0$. Furthermore, the timings of the stimulus bursts are indicated by the segments of the state space in which the muscle was stimulated.

Deviations occur in the tracking of the passive trajectory, because of the nonlinearities in the muscle-skeleton system, which are not described by the simple linear second order model. Muscle dynamics and joint stiffness are particularly nonlinear (AUDU and DAVY, 1985; WiNTERS and STARK, 1987). The effect of the muscle activation can mainly be seen in the fourth quadrant of the figure (negative angle, positive angular velocity). The 

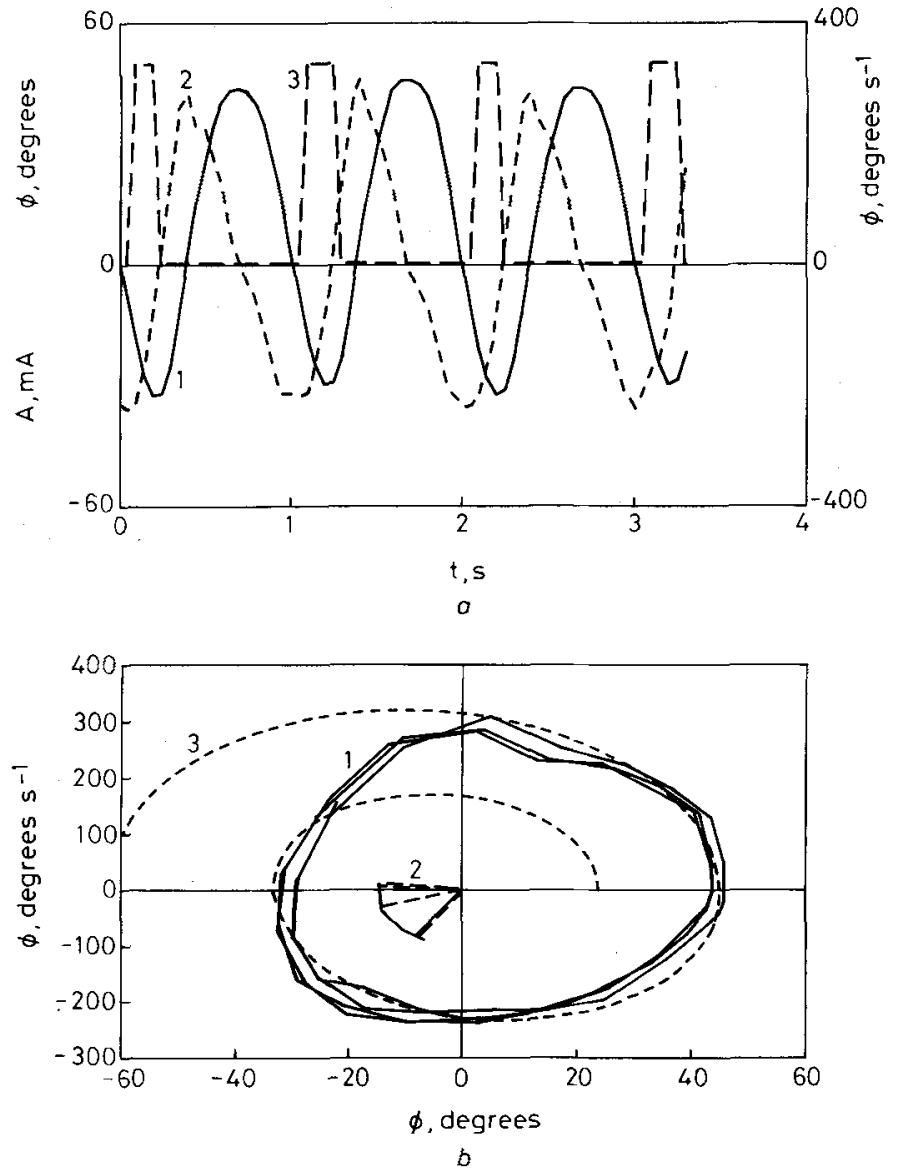

Fig. 6 Recordings of three cycles of the PID-controller trial of Fig. 5a. (a) as a function of time: (1) knee angle $\phi$; (2) knee angular velocity $\dot{\phi}$; (3) stimulus amplitude $A$; (b) in state space: (1) three cycles of the PID-controller trial (Fig. 5a): (2) representation of the stimulation by the segments of the state space in which the quadriceps muscles were stimulated; (3) passive response of a second order linear system $(\zeta=0.08$, cycle time $1.05 \mathrm{~s})$ which satisfies the maximal angle criterion of $45^{\circ}$

stimulation bursts are mainly delivered in the third quadrant. A considerable time difference (about $0.15 \mathrm{~s}$ on average) exists between the end of the stimulation burst and the tracking of the passive state trajectory, which starts at about maximum positive angular velocity.

\section{Discussion}

The time integral of torque criterion, eqn. 1, was analysed in a simulation study for the case of rectangular torque pulses. The criterion appeared to be minimum in the case of the shortest maximum torque pulse. However, several questions on the optimal control of the stimulation remain:

(a) It is not clear how well the minimum time integral of torque corresponds to the minimum muscle energy consumption (CROWNINSHIELD and BRAND, 1981).

(b) What is the influence of the muscle dynamics on the optimal stimulation pattern?

(c) Is muscle fatigue minimum when the energy consumption of the muscle is minimised for these cyclical movements? In the end, the objective is to minimise muscle fatigue, to be able to sustain the cyclical task as long as possible.

The tracking of the passive trajectories in state space (Fig. $6 b$ ) indicates the accuracy of the second order linear model (eqns. 3 and 4) of the passive lower leg system. The model seems accurate enough to serve as the basis for tuning the discrete-time PID-controller.

The discrete time PID-controller appeared to be able to realise the reference angle $\phi_{\max r e f}$, and compensate for the influence of fatigue by adapting the stimulation burst time $T_{\text {on }}$.

In the case of the discrete-time PID-controller, the stimulation during each cycle is delivered open-loop. Only between cycles, the stimulation of the next cycle is determined on the basis of the performances in the previous cycles. If, alternatively, the stimulation is to be controlled during the cycles on the basis of real-time measurements of the state variables joint angle and joint angular velocity, the trajectory in state space has to be predicted over the effective time delay to stop stimulation at the correct moment. A sufficiently accurate model of the muscleskeleton system is needed to perform this prediction. Furthermore, this model has to be adapted continuously for the influence of muscle fatigue, which has to be identified continuously (adaptive control).

The principle of cycle to cycle adaptation of the stimulation patterns on the basis of the performance in previous cycles might be applicable in the control of walking with electrical stimulation. However, it will be more difficult to design rules for the adaptation of the stimulation patterns of the individual muscles on the basis of deviations from conditions in the previous cycles, because the movement is very complex, and the muscle actions interact. Furthermore, changing external circumstances during walking might require fast responses during a cycle.

\section{References}

Audu, M. L. and Davy, D. T. (1985) The influence of muscle model complexity in musculoskeletal motion modeling. $J$. Biomech. Eng., 107, 147-157.

Chizeck, H. J., Kobetic, R., Marsolais, E. B., AbBas, J. J., DonNer, I. H. and Simon, E. (1988) Control of functional neuromuscular stimulation systems for standing and locomotion in paraplegics. Proc. IEEE, 76, 1155-1165.

CrowninshiELD, R. D. and Brand, R. A. (1981) A physiological based criterion of muscle force prediction in locomotion. $J$. Biomechanics, 14, 1981, 793-801.

Marsolais, E. B. and Kobetic, R. (1983) Functional walking in paralyzed patients by means of electrical stimulation. Clinical Orthopaedics, 175, 30-36.

McNeal, D. R., Nakai, R. J., Meadows, P. and Tu, W. (1989) Open-loop control of the freely-swinging paralyzed leg. IEEE Trans. Biomed. Eng., 36, 895-905.

Phillips, C. L. and Nagle, H. T. (1984) Digital control system analysis and design. Prentice Hall, Englewood Cliffs.

Winters, J. M. and STARK, L. (1987) What is gained and what is lost by varying model complexity. Biol. Cybern., 55, 403-420.

YAMAGUCHI, G. T. and ZAJAC, F. E. (1990) Restoring unassisted natural gait to paraplegics via functional nueromuscular stimulation: a computer simulation study. IEEE Trans. Biomed. Eng., 37, 886-902.

\section{Author's biography}

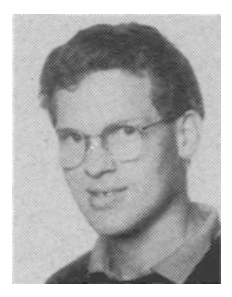

Peter H. Veltink studied Electrical Engineering at the University of Twente, Enschede, the Netherlands. He received the M.Sc. degree in 1984 and the Ph.D. degree in 1988. The topic of his Ph.D. dissertation was 'recruitment of myelinated nerve fibers during artificial nerve stimulation'. In 1989 he visited Case Western Reserve University, Cleveland, where he researched nonlinear control of muscle contraction. Currently, he is member of the Biomedical Engineering Division of the Department of Electrical Engineering, University of Twente. His research is on control of functional neuromuscular stimulation, which is carried out in co-operation with the Roessingh Rehabilitation Center in Enschede. 